\title{
Low Complexity Hybrid PSO-BB Detection Algorithm for Massive MIMO System
}

\author{
M. Kasiselvanathan* and N. Sathish Kumar \\ Department of ECE, Sri Ramakrishna Engineering College, Vattamalaipalayam - 641022, Coimbatore, \\ Tamil Nadu, India; drkasiselvanathanvkm@gmail.com,nsk20022002@gmail.com
}

\begin{abstract}
Objective: A massive Multiple Input and Multiple Output (MIMO) receiver utilizes the proposed detection algorithm to reduce the complexity. Methods: The existing research work namely Noise and Relevancy aware Low Complexity Detection (NRLCD) algorithm for massive MIMO receiver utilizes normalized cross correlation based pruning strategy to viably evacuate uncorrelated signals. However, the existing research work still has more complexity with increasingly number of iterations to find more relevant signal vector. In this research paper, it is proposed to investigate execution of massive MIMO system utilizing Continuous Phase Modulation (CPM) modulation which is used to carry out signal modulation. Then, Hybrid Particle Swarm Optimization-and-Branch-and-Bound (Hybrid PSO-BB) algorithm is proposed for low complexity detection. Findings: CPM demonstrated to give superior performance over Quadrature Amplitude Modulation (QAM) technique with the presence of phase noises. Hybrid PSO-BB is anticipated; in which the best attainable solution were found and renewed using PSO. The performance assessment of the proposed research work and existing methods is done under Adaptive Additive Gaussian Channel (AWGN) using MATLAB Communication tool box. Improvements: From the simulation results, it is inferred that the Hybrid PSO-BB algorithm is superior to the existing methods in-terms of Bit Error Rate (BER) performance and complexity.
\end{abstract}

Keywords: Bit Error Rate (BER), Continuous Phase Modulation (CPM), Low Complexity, Massive Multiple Input and Multiple Output (MIMO), Particle Swarm Optimization (PSO)

\section{Introduction}

Multiple Input and Multiple Output (MIMO) technology improves the capacity of wireless networks and the reliability of data transmission through wireless media networks ${ }^{1-3}$. Single-Input Single-Output (SISO) technology uses the only one transmitter and one receiver to support limited wireless services of upper information rates. To achieve high transmission rate, SISO technology must have large bandwidth and signal to noise ratio ${ }^{4}$. Massive MIMO uses large antennas at both ends and provides better performance without requiring additional bandwidth or traditional power ${ }^{5}$. A Random Restart RTS (R3TS) algorithm was proposed to attain the improved BER performance when it is compared with the traditional RTS algorithm using higher-order QAM modulation ${ }^{6}$.
PRUNing based Maximum Likelihood Detection using Low Complexity Detection Algorithm (PRUN-MLD -LCDA) is used to find the more relevant signal vectors based on the cross correlation based pruning technique ${ }^{7}$.

CPM modulation has constant envelope signals with good power and spectral efficiency ${ }^{8}$. It is having these favourable features, but still it is limited to have low complexity ${ }^{9}$. To reduce the complexity in the received signals, some key features are performed to introduce the CPM signals with Rimoldi decomposition and Walsh decomposition 10,11 . In both Rimoldi decomposition and Walsh decomposition, number components have been reduced to represent the signal space and it these decomposition methods do not reduce the number of states. The Laurent decomposition is introduced to allow decomposing the CPM signal as a superposition of

*Author for correspondence 
linearly modulated signals ${ }^{12}$. The Laurent decomposition method reduces the number of states required to describe the CPM signal. This method is commonly applied in the literatures to design low complexity MIMO receiver and also used to derive the data aided frequency detectors $\underline{\underline{13}}-\underline{15}$. Binary CPM modulation with 0.5 modulation index was used to give low complexity detector and is approximated by linear modulation method. The soft decision decoding was produced for ceded CPM system and detector gives low complexity.

The study is formulated as follows. The Section 2 describes the massive MIMO system model and CPM modulation in detail. The proposed MIMO receiver algorithm is dealt in section 3. The simulation results are depicted in section 4 which summarizes the BER performance comparison and computational complexity. Section 5 is dealt about conclusion which infers that the BER performance of Hybrid PSO-BB provides significantly outperforms than the conventional methods.

\section{System Model}

The system model is given in Figure 1. The CPM modulator is used to generate the transmitted signal $\mathrm{S}(\mathrm{t}, \mathrm{a})$ given the $\mathrm{M}$-ary information sequence $\mathrm{a}=\left\{\mathrm{C}_{0}, \mathrm{C}_{1}, \ldots, \mathrm{C}_{\mathrm{N}-1}\right\}$, and the $\mathrm{CPM}$ signal which has complex values is defined as:

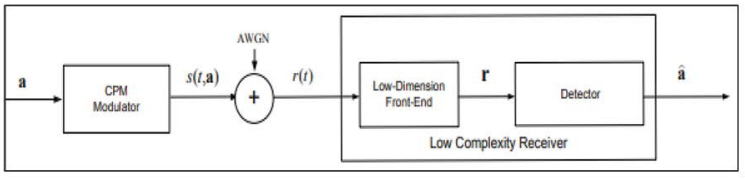

Figure 1. System model.

$$
S(t, a)=\sqrt{\frac{2 E_{s}}{T}} \exp \left\{j 2 \pi h \sum_{i=0}^{n} a_{i} q(t-i T)\right\}
$$

Where $E_{s}$ is the energy per symbol, $T$ represents symbol interval, the modulation index is represented by $\mathrm{h}=\mathrm{k} / \mathrm{p}$ where $\mathrm{k}$ and $\mathrm{p}$ denoted as prime integers. The symbols $a_{i}$ are assumed to be independent and takes the values $\{ \pm 1, \pm 3, \ldots, \pm(\mathrm{M}-1)\}$ from the $\mathrm{M}$-ary alphabet. The function $q(t)$ is denoted as phase response and its derivative is frequency pulse with the duration L.

The information bearing phase $\theta(t, a)$ is defined accordingly as:

$$
\begin{aligned}
& \theta(t, a)=2 \pi h \sum_{n=0}^{k} a_{n} q(t-n T)=\pi h \sum_{n=0}^{k-L} a_{n} \\
& +2 \pi h \sum_{k=N-L+1}^{k} a_{n} q(t-n T)=\theta_{n}+\theta(t) \\
& \theta_{n} \triangleq\left[\pi h \sum_{i=0}^{n-L} a_{i}\right] \bmod 2 \pi
\end{aligned}
$$

$$
\theta(t) \triangleq 2 \pi h \sum_{i=n-L+1}^{n} a_{i} q(t-i T)
$$

Where $\theta_{n}$ is the accumulated phase and $\theta(t)$ is the incremental phase within one interval. At the receiver side, coherent trellis defined accordingly as shown in Figure.1.

In this work, we focus on the AWGN channel wherein the received signal $r(t)$ reads:

$$
r(t)=S(t, a) e^{j \Phi_{n}}+n(t)
$$

Where, assume $\Phi_{n} \bmod 2 \pi \in[0,2 \pi]$ is the random. $n(t)$ is denoted by white Gaussian noise with zero mean with the power spectral density of $\mathrm{N}_{\mathrm{o}} / 2$. The phase noise is modeled as a discrete time random walk (Wiener) process defined as:

$$
\Phi_{n}=\Phi_{n-1}+\sigma_{n}
$$

Where $\Phi_{n}$ is assumed as independent and identically (i.i.d) Gaussian random variable. Then we define the normalized Euclidean distance in $\mathrm{Eq}(7)$.

$$
d^{2}(a, b)=\frac{1}{2 E_{b}} \int_{0}^{N T}|S(t, a)-S(t, b)|^{2} d t
$$

$d_{\min }^{2}={ }_{a \neq b}^{\min } d^{2}(a, b)$

Where, $E_{b}$ is the average energy per information bit. $\mathrm{CPM}$ is widely used modulation scheme and it is energy efficient because CPM signals can be transmitted using nonlinear amplifiers. The modulation scheme must be efficient and to be designed to meet the needs as the date rate increases. Linear modulation schemes like QAM are 
more efficient in-terms of spectral bandwidth than CPM. But QAM signals are not energy efficient as they have transmitted by linear amplifiers. Hence communication system has to be designed using an energy efficient modulation scheme. In this research we consider CPM modulation which is energy efficient than QAM.

\section{Hybrid PSO-BB Detection Algorithm}

The existing techniques tend to have optimization difficulty which would increase the complexity. This can be overcome by introducing proposed Hybrid PSO-BB algorithm to ensure low complexity detection at the receiver. The proposed method produces the optimal value by giving solution for linear programming relax for Lower Bound (LB) and the best possible value for Upper Bound (UB) and renewed with PSO. PSO is applied to MIMO technology for providing faster convergence and lower overall complexity. Given input signals are updated by the best score of PSO algorithm. Fitness function is error rate of the actual and predicted signal.

\section{Algorithm Steps:}

Step 0: Set $A=0, \Omega=\{P\}$. // Initialize

Find the optimal value by giving solution for linear programming relax for $\mathrm{LB}$ and the value for UB and best feasible value $\mathrm{Y}$ of the problem (ISCCP) with PSO.

Step A $(\mathrm{A}=1,2,3, \ldots)$

A1: if $\Omega=\varnothing$ or UB-LB/UB<eps, the the outcome $\mathrm{Y}$ and $\mathrm{OPT}=\mathrm{UB}$. // Terminate.

A2 : find sub-rectangle $\mathrm{P}_{\mathrm{K}}$ in $\Omega$ such that $\mathrm{LB}\left(\mathrm{P}_{\mathrm{K}}\right)=\mathrm{LB}$. // Selection Rule.

A3 : Partition $P_{K}$ into two sub-rectangles $P_{K+1,1}, P_{K+1,2}$. Then each sub-rectangle

is reduced into integer sub-rectangle. The obtained sub-rectangle are denoted as

$$
\mathrm{P}_{\mathrm{K}+1,1}, \mathrm{P}_{\mathrm{K}+1,2} \text { too. Set } \Omega=\left(\Omega-\mathrm{P}_{\mathrm{K}}\right)\left\{\mathrm{P}_{\mathrm{K}+1,1}, \mathrm{P}_{\mathrm{K}+1,2}\right\} . / /
$$
Branching.

A4: Get new Lower LB to solve the problem over $\mathrm{P}_{\mathrm{K}+1,1}$ $\& \mathrm{P}_{\mathrm{K}+1,2}$ respectively.

//Lower Bounding

A5 : UB and Y are renewed with PSO. // Upper Bounding

A6 : Set $\Omega=\Omega-[\mathrm{P} \in \Omega)$ : LB $(\mathrm{P})>=\mathrm{UB}]$

$\mathrm{A}=\mathrm{A}+1$. Go to A1. // Deleting Rule

\section{Results and Discussion}

The simulations results are carried out by using the metric called BER. The performance analysis is done using MATLAB Communication tool box version 13a under AWGN channel for $(32 \times 32)$ and $(64 \times 64)$ transmit and receive antennas with CPM modulation with 0.5 modulation index. Figure 2 shows the BER performance of the Hybrid PSO-BB detection using CPM (CPM-PSO), NRLCD detection and PRUN-MLD-LCDA detection for $(32 \times 32)$ and $(64 \times 64)$ antennas at both base station and user terminal. From the Figure 1, it is inferred that the proposed research methods tends to have better performance in terms of lesser BER rate than the other methods. The number of arithmetic operations used by an algorithm gives computational complexity.

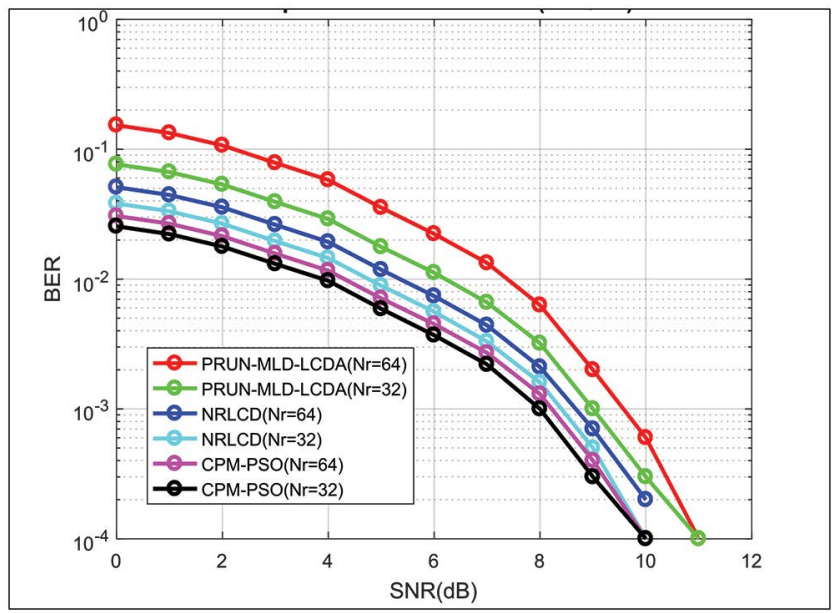

Figure 2. BER performance comparison of detection methods.

Table 1 shows the computational complexity performance comparison of proposed method using CPM modulation scheme and existing methods. Table 1, the Hybrid PSO-BB detection algorithm gives less complexity than the existing detection algorithms. Table 2 shows the time complexity performance comparison of proposed method using CPM modulation scheme and existing methods for varying the message sizes from 100 to 500. From Table 2, Hybrid PSO-BB detection algorithm gives lesser time complexity where it is $22 \%$ better than NRLCD detection algorithm, 27\% lesser time complexity than PRUN-MLD-LCDA algorithm. 
Table 1. Computational complexity comparison of detection algorithms

\begin{tabular}{|c|c|c|c|c|}
\hline \multirow{2}{*}{ Detection Methods } & \multicolumn{4}{|c|}{ Number of Arithmetic Operations } \\
\cline { 2 - 5 } & Addition & Subtraction & Multiplication & Division \\
\hline PRUN-MLD-LCDA & 354 & 195 & 460 & 119 \\
\hline NRLCD & 265 & 210 & 436 & 131 \\
\hline Hybrid PSO-BB with CPM & 289 & 181 & 485 & 127 \\
\hline
\end{tabular}

Table 2. Time complexity comparison of detection algorithms

\begin{tabular}{|c|c|c|c|c|c|}
\hline \multirow{2}{*}{ Detection Methods } & \multicolumn{5}{|c|}{ Time Complexity (ms) } \\
\cline { 2 - 6 } & $\mathbf{1 0 0}$ & $\mathbf{2 0 0}$ & $\mathbf{3 0 0}$ & $\mathbf{4 0 0}$ & $\mathbf{5 0 0}$ \\
\hline PRUN-MLD-LCDA & 18 & 20 & 26 & 31 & 35 \\
\hline NRLCD & 15 & 17 & 25 & 27 & 29 \\
\hline $\begin{array}{c}\text { Hybrid PSO-BB } \\
\text { with CPM }\end{array}$ & 12 & 17 & 20 & 23 & 29 \\
\hline
\end{tabular}

\section{Conclusion}

The Hybrid PSO-BB detection algorithm is proposed for massive MIMO system to detect the received signals with low complexity. The proposed method produces the optimal value by giving solution for linear programming relax for $\mathrm{LB}$ and the best possible value for $\mathrm{UB}$ and renewed with PSO. This algorithm is anticipated; in which lower bound of ideal values was determined using linear programming and upper bound of optimal value and the best attainable solution were found and renewed using PSO. The proposed algorithm uses a CPM modulation with the modulation index of 0.5 . The performance analysis is carried out under AWGN for large number of transmitting and receiving antennas. From the simulation results, it is inferred that the Hybrid PSO-BB algorithm is superior to the existing methods in-terms of BER performance and complexity.

\section{References}

1. Chockalingam A, Lakshmi Narsimhan, T. Channel Hardening Exploiting Message Passing (CHEMP) receiver in Large Scale MIMO systems, IEEE Transaction on Information Theory. 2014; 8(5):847-60. https://doi. org/10.1109/JSTSP.2014.2314213.
2. Rajan BS, Chockalingam A, Mohammed SK, Vardhan KV. A low complexity detector for large scale MIMO syste and multi carrier CDMA systems, IEEE Journal of Areas in Communications. 2008; 26(3):473-85. https://doi. org/10.1109/JSAC.2008.080406.

3. Chockalingam A, Mohammed SK, Rajan BS, Zahi A. High rate space time coded large MIMO systems: Low complexity detection and channel estimation, IEEE Journal Selected Topics in Signal Processing. 2009; 2(6):958-74. https://doi. org/10.1109/JSTSP.2009.2035862.

4. Badjate SL, Raut PW. MIMO-Future wireless communications, International Journal of Innovative Technology and Exploring Engineering. 2013:2(5):102-6.

5. Viterbo E, Cerato B. Hardware implementation of a low complexity detector for large MIMO, Proceedings of IEEE International Symposium on Circuits and Systems (ISCAS'09). 2009; 593-6.

6. Srinidhi N, Datta T, Chockalingam A, Rajan BS. Random-restart reactive tabu search algorithm for detection in large MIMO systems, IEEE Communications Letters. 2010; 14(12):1107-09. https://doi.org/10.1109/ LCOMM.2010.101210.101587.

7. Kasiselvanathan M, Sathish Kumar, N. Bit error rate aware accurate prediction of original signals with low distortion using low complexity detection algorithms, Concurrency Computation and Practice Experience. 2019. https://doi. org/10.1002/cpe.5192.

8. Aulin T, Anderson JB, Sundberg CE. Digital Phase Modualtion. New York, USA: Plenum; 1986. https://doi. org/10.1007/978-1-4899-2031-7.

9. Simmons SJ, Wittke, PH. Low complexity decoders for constant envelope digital modulations, IEEE Transaction on Communication. 1983; 31(12):1273-80. https://doi. org/10.1109/TCOM.1983.1095778.

10. Rimold BE. A decomposition approach to CPM, IEEE Transaction on Information Theory. 1988; 34(2):260-70. https://doi.org/10.1109/18.2634.

11. Shwedyk E, Tang W. A quasi optimum receiver for Continuous phase modulation, IEEE Transaction on Communication. 2000; 48(7):1087-90. https://doi. org/10.1109/26.855514. 
12. Laurent PA. Exact and approximate construction of digital phase modulations by superposition of Amplitude Modulated Pulses (AMP), IEEE Transaction on Communication. 1986; 34(2):150-60. https://doi. org/10.1109/TCOM.1986.1096504.

13. Kaleh GK. Simple coherent receivers for partial response continuous phase modulation, IEEE Journal Selected Areas in Communications. 1989; 7(9):1427-36. https://doi. org/10.1109/49.44586.
14. Andrea, AN, Ginesi, A, Engali U. Frequency detectors for CPM signals, IEEE Transaction on Communication. 1995; 43(2-4):1828-37. https://doi.org/10.1109/26.380234.

15. Xi z, Zhu J, Fu, Y. Low complexity detection of binary CPM with small modulation index, IEEE Communication Letters. 2016; 20(1):57-60. https://doi.org/10.1109/ LCOMM.2015.2503761. 